\title{
WS9-B04
}

\section{Estimation of the Petrophysical Model through the Joint Inversion of Seismic and EM Attributes}

\author{
F. Miotti* (Schlumberger), I. Guerra (Schlumberger), F. Ceci \\ (Schlumberger), A. Lovatini (Schlumberger), M. Paydayesh \\ (Schlumberger), G. Milne (Schlumberger), M. Leathard (Schlumberger) \& \\ A. Sharma (Schlumberger)
}

\section{SUMMARY}

Reservoir characterization objectives are to estimate the petrophysical properties of the prospective hydrocarbon traps and to reduce the uncertainty of the interpretation. In this framework, we present a workflow for petrophysical joint inversion (PJI) of seismic and EM attributes to estimate the petrophysical model in terms of porosity and water saturation. This study realizes the joint inversion within the probabilistic structure provided by the Bayesian theory. 3D volumes of estimated porosity and saturation show how the joint inversion of acoustic impedance and electrical resistivity can provide a quantitative description of the reservoir properties and with it a measure of uncertainty, which is consistent with the petrophysical model and observations. 


\section{Introduction}

Reservoir characterization objectives are to estimate the petrophysical properties of the prospective hydrocarbon traps and to reduce the uncertainty of the interpretation. In this framework, we present a workflow for petrophysical joint inversion (PJI) of seismic and EM attributes to estimate the petrophysical model in terms of porosity and water saturation. This study realizes the joint inversion within the probabilistic structure provided by the Bayesian theory. 3D volumes of estimated porosity and saturation show how the joint inversion of acoustic impedance and electrical resistivity can provide a quantitative description of the reservoir properties and with it a measure of uncertainty, which is consistent with the petrophysical model and observations.

\section{Method}

According to the rock cross property concept (Carcione et al., 2007), for integrating heterogeneous measurements, we must define some constitutive equations that link rock properties with well-log measurements. Many rock physics models are available in the literature and their efficacy depends on the particular lithology of the sediments (Schön 1996). Also in 3D model scale, the rock physics modeling allows the estimation of the petrophysical parameters. We analyze a reservoir scenario, where porous sandstone is saturated with oil, water and gas. As data input PJI uses the seismic attributes, derived from the AVO inversion, (acoustic impedance-AI, density and Poisson's ratio-PR) together with the resistivity model resulting from the CSEM inversion. All models are defined within the same grid in order to have the same number of cells. The CSEM inversion produces a lowresolution model with respect to the seismic attributes. We mitigate this limitation exploiting the transverse resistance principle, (Constable 2010). For modeling the relation $d=g(m)$, we follow the Tarantola's approach on inverse problems, (Tarantola, 2005). The vector $m$ defines the unknown model parameters, (porosity and water saturation in the bi-phase configuration), while the $d$ vector represents the input data, (acoustic impedance and electrical resistivity). According to the Bayesian theory, the state of information on the model parameters is described by the prior model $m_{\text {prior }}$ and by $\mathrm{C}_{\mathrm{M}}$, the covariance matrix that takes into account its uncertainties. The uncertainty associated with the observed data is captured by $\mathrm{C}_{\mathrm{D}}$, (data covariance matrix). We assume Gaussian probability distribution for both model parameters and data. The solution of the inverse problem is obtained through an iterative procedure that linearizes the forward model around the current model $m_{k}$, and obtains a new model $m_{k+1}$. The closed-form solution is:

$$
\left.m_{k+1}=m_{\text {prior }}-\left[G_{k}{ }^{T} C_{d}{ }^{-1} G_{k}+C_{M}{ }^{-1}\right]^{-1} G_{k}{ }^{T} C_{d}{ }^{-1} \cdot \mid\left(g\left(m_{k}\right)-d\right)-G_{k}\left(m_{k}-m_{\text {prior }}\right)\right]
$$

Further, the algorithm provides the model posterior covariance matrix $C_{M, p o s t}$, which describes the uncertainty of the solution as: $C_{M, \text { post }}=\left(G_{k}{ }^{T} C_{d}{ }^{-1} G_{k}+C_{M}{ }^{-1}\right)^{-1}$. The sensitivity analysis and regularization of the inverse procedure are both detailed by Dell'Aversana et al., (2011).

\section{Example}

The PJI technology was tested on a real exploration case. The input data consists of seismically derived AI, density and CSEM derived electrical resistivity attributes. The PJI objective is to describe quantitatively the petrophysical properties of a potential reservoir, in terms of porosity and water saturation distribution within the 3D model by using the rock physics model framework. We focus within a selected area where both seismic attributes and resistivity models show correlated anomalies as evidence of a potential reservoir (Guerra et al., 2013). The joint anomalies are depicted in Figure 1 (left). We notice a low PR and high resistivity values in the investigated region. Because well logs are not available in this area, we calibrated the priori rock model through the analysis of the scatter plots into rock model templates. To test the contribution of the electrical resistivity, two tests are performed. In the first we use as input AI and density model (Petrophysical Inversion - PI), while in the second AI and electrical resistivity model (Petrophysical Joint Inversion - PJI). The comparison is 


\section{Amsterdam ' 14}

in Figure 1 (right), which shows the porosity and water saturation models on the horizontal z-slice at a depth of $-967 \mathrm{~m}$. Both porosity estimates A and, D are consistent because this rock property is mainly derived by the $\mathrm{AI}$ attribute. In contrast, the comparison between $\mathrm{B}$ and $\mathrm{C}$ exhibits the role of the resistivity attribute to discriminate fluids, (e.g., water from gas/oil). Model B is a "smooth" water saturation model because density and AI attributes are weakly sensitive to fluid substitution watergas. For this reason the model shows several values close to the priori water saturation, (set to 0.5). The model $\mathrm{C}$ highlights the high sensitivity of resistivity with respect to resistive fluids (oil and/or gas). The PJI strongly reduces the amount of water saturation corresponding to the resistive anomaly as evidence of a possible presence of gas saturation, which is the preliminary assumption we are supporting, (Guerra et al., 2013). Because no well logs are available in this survey area, the results assessment is based on cooperative interpretation based on cross plot analysis.
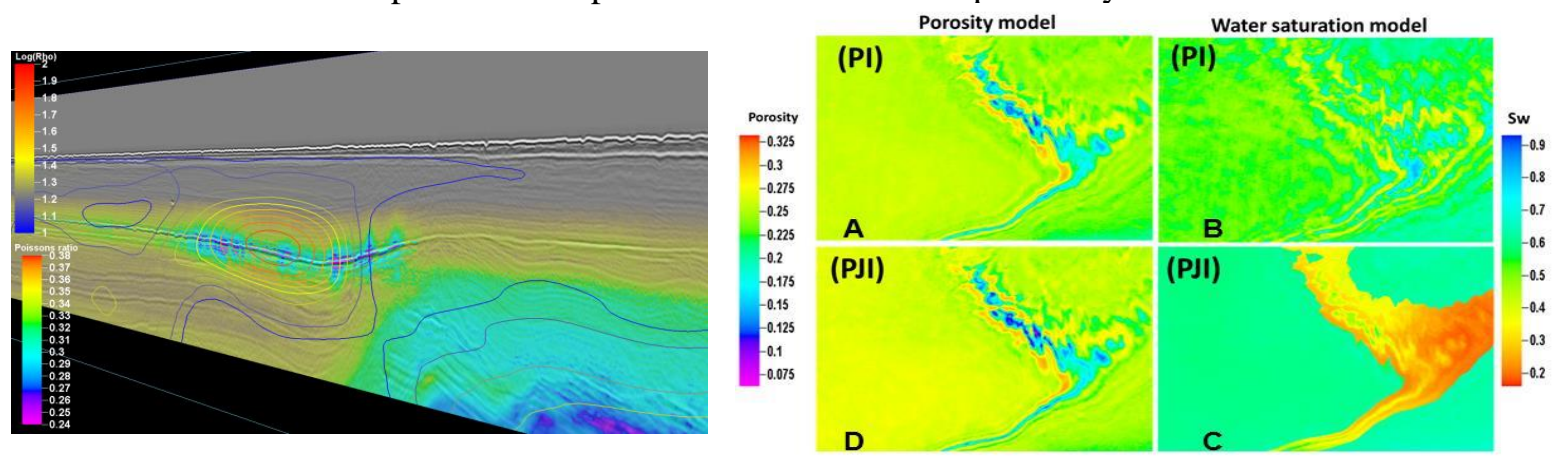

Figure 1: left) Seismic data co-rendered with $P R$ and resistivity (contour lines). Right) $A$ and $B$ are the porosity and water saturation models resulting from the PI. D and $C$ are the porosity and water saturation model resulting from PJI. C model shows the EM contribution to discriminate the water content from resistive fluids (oil and/or gas).

\section{Conclusion}

The results evidence the contribution of the joint inversion of seismic and EM attributes to derive a quantitative description of the petrophysical model. This study supports the role of the CSEM technology in conjunction with seismic attributes to reduce uncertainty in prospect generation. Once prospects are identified, (with complimentary structural, stratigraphic and DHI's with CSEM resistivity indicators) the PJI is used as a quantifier for reservoir attributes. Finally, it is highly recommended to use well log data to better calibrate a representative rock model for the survey area, in order to improve the final result.

\section{Acknowledgements}

The authors thank Schlumberger and EMGS for allowing access to their datasets.

\section{References}

Carcione J.M., Ursin B. and Nordskag J.I. 2007. Cross-property relations between electrical conductivity and the seismic velocity of rocks. Geophysics 72, 193-204.

Constable S. 2010. Ten years of marine CSEM for hydrocarbon exploration. Geophysics, 75, No 5. PP75A67-75A81.

Dell'Aversana P., Bernasconi G., Miotti F. and Rovetta D. 2011. Joint inversion of rock properties from sonic, resistivity and density well-log measurements. Geophysical Prospecting 59, 1144-1154.

Guerra I., Ceci F., Miotti F., Lovatini A., Milne G., Paydayesh M., Leathard M., Sharma A., Panzner M. and Gabrielsen P., 2013. Multi-measurement integration - a case study from the Barents Sea. First Break, 31, 55-61.

Schön J.H. 1996. Physical Properties of Rocks: Fundamentals and Principles of Petrophysics. Pergamon Press. ISBN 10008044346X.

Tarantola A. 2005. Inverse Problem Theory. SIAM. ISBN9780898715729. 